\title{
Fractal dimension as tool for vascular diagnosis in health
}

José Guedes da Silva Júnior"* , Iasmyn Dayanne Santos do Nascimento², Sílvia Renata Ribeiro Araújo ${ }^{3}$, Camila Geórgia Silva Nascimento ${ }^{3}$, Maria Eduarda Barbosa de Moraes ${ }^{3}$, Layslla Caroline Araújo Almeida ${ }^{3}$, Mônica Nayara Batista Barbosa ${ }^{3}$, Hallysson Douglas Andrade de Araújo ${ }^{1}$, José Adelson Alves do Nascimento Júnior ${ }^{1}$, Nadine Gabryella Pontes Maciel ${ }^{1}$, Victor Felipe da Silva Araújo ${ }^{4}$, Milena Mácia da Silva ${ }^{1}$ and Talyta Valéria Siqueira do Monte ${ }^{5}$

${ }^{1}$ Universidade Federal de Pernambuco, Brazil

${ }^{2}$ Centro Universitário Saão Miguel, Brazil

${ }^{3}$ Centro Universitário Mauricio de Nassau, Brazil

${ }^{4}$ Universidade Federal Rural de Pernambuco, Brazil

${ }^{5}$ Hospital Otávio de Freitas, Brazil

\begin{abstract}
Cancer is a pandemic disease, and can affect anyone regardless of age or gender. In Brazil, the estimated cancer incidence, released by the National Cancer Institute José de Alencar (INCA), estimates that the country should register about 600 thousand new cases of cancer per year in 2018 and 2019. However, data released by WHO (World Health Organization) in 2018, claim that about 9.6 million people die each year from cancer, and one in six deaths are related to the disease. Researchers worldwide seek the cure of the most diverse types of cancer, molecular markers, prophylactic treatments and faster and safer diagnostic methods. One of the major barriers to the cure of cancer is still metastasis, in this case, the formation of new blood vessels, their growth and the way it expands in the patient's body. The fractal methodology comes as a way of assisting in the diagnosis and monitoring of the evolution of cancer and consequently of its treatment. Measuring progression, delay or paralysis of metastasis.
\end{abstract}

\section{Introduction}

Malignant neoplasms are after cardiovascular diseases, the second most common cause of death in industrialized countries, and metastatic dissemination is responsible for failure of therapy and prognosis [1]. According to data released by PAHO [2] and WHO [2] in 2018, about 9.6 million people die each year from cancer, one in six deaths are related to the disease. Being more than $70 \%$ of these deaths in low or medium developing countries. About one-third of cancer deaths are due to five major behavioral and eating risks: high body mass index, low fruit and vegetable intake, lack of physical activity and use of alcohol and tobacco. Smoking is the main risk factor for cancer, causing $22 \%$ of deaths from the disease. In Brazil the most frequent cancer is nonmelanoma skin, with the second position occupied by breast cancer in women and prostate cancer in men. In the estimate of the incidence of Cancer in Brazil, published by the National Institute of Cancer José de Alencar, it is estimated that the country should register about 600 thousand new cases of cancer per year in 2018 and 2019 [3].

Cancer is a generic term for a large group of diseases that can affect any part of the body. Other terms used are malignant tumors and neoplasms. A characteristic that defines cancer is the rapid proliferation of abnormal cells that grow beyond their usual limits in a disordered way and can invade adjacent parts of the body and spread to other organs and tissues, a process referred to as metastasis. Metastasis occurs through blood or lymphatic migration is the leading cause of cancer death [3]. When some genes that express proteins with actions related to the control of the cell cycle undergo mutations, the cells begin to undergo alterations of their normal state and assume compatible characteristics of a neoplastic cell. Genes responsible for stimulating cell division are generally referred to as oncogenes and if they have the function of inhibiting it, they will be considered as tumor suppressor genes. Either by an abnormally exacerbated function of the oncogenes or by suppressor inhibition, the result will be the obtaining of a cell that will present a proliferative gain in relation to the others, becoming insensitive to the apoptotic stimuli and end up tending to be very aggressive, determining the formation of tumors (accumulation of cancerous cells) or malignant neoplasms [3].

Cancer derives from changes and some physiological changes in the cell are fundamental for the disease to manifest, have a great genetic variability and a large set of factors that can lead to its appearance or its proliferation. There are changes that can be defined as the main characteristics that are common in the types of cancers $[4,5]$. Such changes may be either intrinsic, such as inherited genetic mutations or random errors in DNA replication, or extrinsic, for example: damage and genetic instability induced by radiation, by chemicals or by viral infection. A single mutation, however, hardly leads to tumor formation; for this process to occur, two to six (or more) genetic modifications are required, which will progressively interfere with the mechanisms responsible for proliferation, differentiation and cell death [6].

In their review, Hanahan and Weinberg [7,8] suggested six fundamental changes in cell physiology that would govern the transformation of a normal cell into a cancer cell: self-sufficiency in signs of proliferation, insensitivity to growth inhibitory signals, evasion of apoptosis, replication potential unlimited, sustained angiogenesis, and tissue invasion and metastasis. Each of these "competencies", which can

*Correspondence to: José Guedes da Silva Júnior, Universidade Federal de Pernambuco - UFPE, Recife, Pernambuco, Brazil, E-mail: juniorguedes18@ hotmail.com

Key words: cancer, fractal, diagnosis, innovation

Received: July 09, 2019; Accepted: July 22, 2019; Published: July 26, 2019 
be achieved through different molecular mechanisms, would represent the overcoming of different strategies, intrinsic to cells and tissues, against tumor development, and the combination of these alterations would aid in the growing malignancy and progression of cancer.

A tumor is characterized by tissue enlargement, that is, a marked cell multiplication and for the same to be considered detrimental to the individual needs to have a very prominent vascularization with respect to the normal tissues [4] therefore the malignant tumor growth and its progression until the development of metastasis depend on a crucial step: angiogenesis, which by definition is the process of formation and growth of new blood vessels. This ability is not unique to cancer, because other diseases are characterized by their angiogenic capacity, in addition, angiogenesis is present since the embryonic development. It is a crucial phenomenon in fetal growth, from the formation of the placenta to the branch of the child's circulatory system. In the adult it is necessary in the repair or regeneration of the tissue, in the process of homeostasis and wound healing. In women, angiogenesis is active at the uterine level for a few days of the menstrual cycle, as well as ovulation and maturation of oocytes. Normal physiological angiogenesis is characterized by being a regulated process with limited duration $[9,10]$. Although the starting point of the genetic alteration occurs in the cell, it is now known that only the cellular study is not enough. The perception of cancer as a tumor microenvironment, which interacts with genetically altered cells, normal cells (such as fibroblasts, immune cells, endothelial cells), vessels, and substances produced locally or from blood supply, has been shown to be much more satisfactory in understanding development of the tumor [5]. In fact, recent studies show that non-cancer cells have a significant role in various tumor progression processes, such as angiogenesis, metastasis, and cell proliferation. Thus, the tumor can be seen as a complex tissue, with distortion of the original tissue homeostasis, and in which "normal" cells are coopted to function according to this new tissue dynamics, mainly dictated by the cancerous cells [6].

Regarding the diagnosis of the disease, if there are prophylactic measures, such as self-examination, used for the diagnosis of breast or prostate cancer, genetic mapping is used to look for genetic alterations that may lead to benign or malignant tumors. In addition, cytopathological examinations are performed for the best cellular visualization of the affected tissues, as well as imaging examinations, where it is possible to visualize the tumor mass. In the scientific scope studies advance with the expectation of finding tumor markers in routine examinations that can serve for a greater agility in the diagnosis and consequently, in the treatment.

Cancer is a pandemic disease, and can affect anyone regardless of age or gender. Researchers from all over the world are waging a battle, both in the agility of the diagnosis, and in the efficiency of the treatment applied to the patient. In this way, new lines of research appear at all times proposing, therapeutics, more precise and specific diagnostics and complementary monitoring methods. Therefore, the objective of the present study is to demonstrate how fractural dimensions, mathematical methods, which are little explored in daily routine examinations, could help in the complementary diagnosis and in the therapeutic monitoring of neoplasms.

\section{Fractal dimension}

In 1975 Benoit Mandelbrot, a French natural mathematician from Poland, made use of the term fractal, to describe the geometry that sought to represent the real forms of nature, where the term fractal derives from Latin for fractals meaning break or irregular. Therefore,

fractals are objects generated by the repetition of a recursive process (its geometric form), presenting autosimilarity, complexity and fractional dimension each time in smaller scales [11]. Fractals are marked by the following peculiarities: autosimilarity, which means that parts of an object or process have statistical similarities at various scales; scaling, which means that the measure of magnitude varies according to the scale that was used; fractal dimension, which provides a quantitative description of autosimilarity and scale submission and the irregular statistical properties of fractal magnitudes [12,13] (Figure. 1).

In the case of the frequent construction of a mathematically de fi ned fractal, there is an infinite number of procedures to be executed, thus generating an infinitely complex structure. Finally, the size of a fractal, unlike Euclidean geometry, is not necessarily an integer value. In it, a point has dimension zero, a line has dimension one, a surface has dimension two and a volume has dimension three. In the case of the fractal dimension, it is a fractional quantity, representing the degree of occupation of the structure in the space that contains it [13,14] (Figure. 2).

The concept of fractal dimension is usually applied and calculated for figures, lines or surfaces in the mathematical and physical branch, but it is currently also gaining space in medicine being used as an auxiliary method in the diagnosis of diseases. One of the fields where this procedure has been developed is the diagnosis of cancer through the analysis of tumor images. Experimental evidence suggests that cancer tumors have a fractal dimension borderline than those occurring in normal tissue aggregates [15] (Figure. 3).

Malignant tumors have a remarkable vascularity compared to normal tissues already established. These findings were considered as consequences of the inflammatory process due to the areas of necrosis in the tumor mass. However, there is currently evidence that the

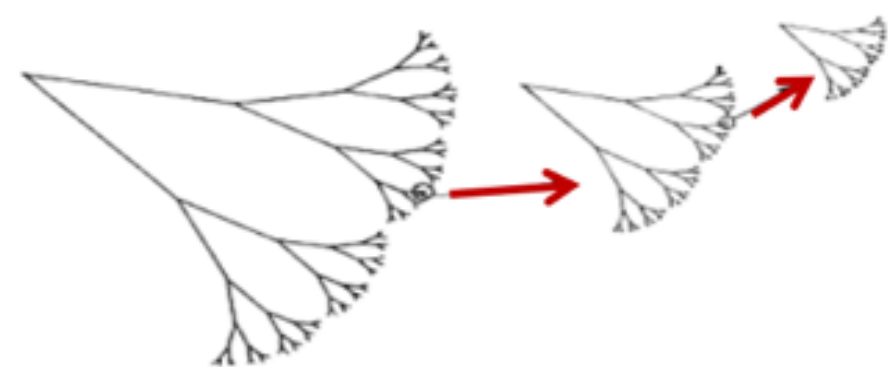

Figure 1. Example of a fractal structure constructed iteratively depicting the characteristic of self-similarity. The construction of this structure starts with a ribbon with a given length and provided with a certain width. The upper half is replaced by two branches with half as much length and width, with the "branches" always forming the same angle. This process continues until a fractal in the shape of a tree is generated. For infinite progressions, the complexity of the structure (13) is verified
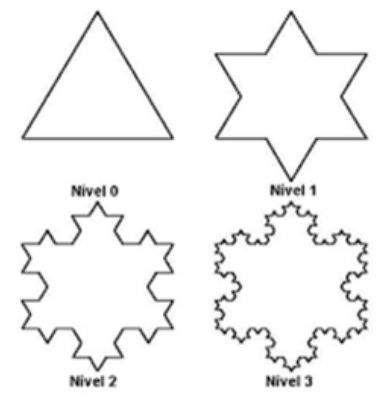

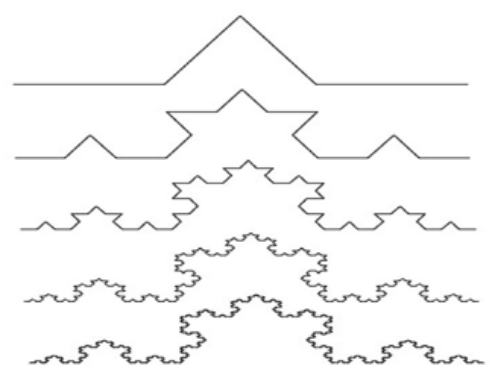

Figure 2. Examples of fractal structures that show self-similarity, its infinite progressions and complexity of the most varied structures 


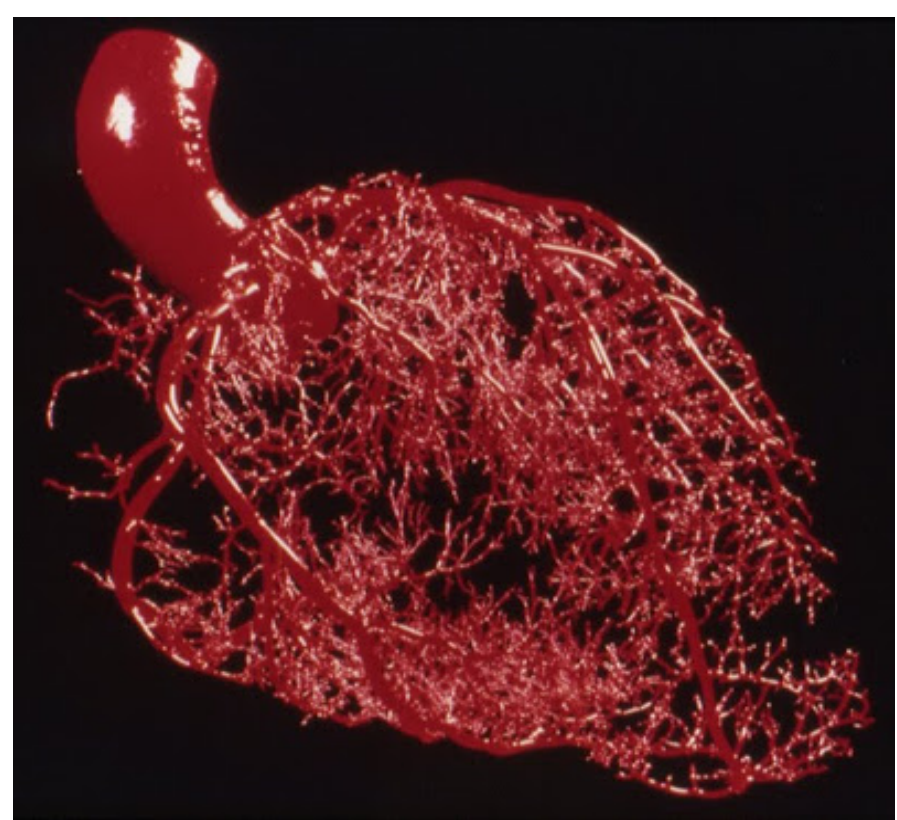

Figure 3. Cardiovascular arterial system in fractal dimensions, exemplifying the use of the mathematical method in biological use

presence of this intense vascularization is an essential condition for neoplastic development [4]. This is due to the development of micro vessels from endothelial cells belonging to capillaries located near the neoplastic cells. The capillaries are blood vessels of small size, being present throughout the body and its extension equals $1000 \mathrm{~m}^{2}$, these are formed by endothelial cells that can be considered among those that present a low rate of cellular divisions between the other cells of the body, with a proliferative cycle comprising hundreds of days, while epithelial cells, for example, can divide up to twice daily [4].

With this, the intense vascularization in a tumor tissue demonstrates two very relevant aspects. The first of these is the existence of the stimulus for these endothelial cells, capable of altering their normal state of division. The second is the local nature of this stimulus, since such division will occur only in the segment adjacent to the emergence of a tiny clone of neoplastic cells. Where we know that this vascular growth is called angiogenesis $[4,16]$.

Judah Folkman proposed in 1971 that the growth of solid neoplasms is always accompanied by neovascularization and this new capillary growth is still more vigorous and continuous than a similar growth of capillary shoots observed in fresh wounds or in inflammation. At that time Folkman already suggested that it would be of great importance a line of studies in the antineoplastic therapy being directly related to the development of techniques capable of blocking or inhibiting the emergence of this neovascularization, in order to prevent tumor growth [17]. Considering that with the progression of metastasis we have a simultaneous progression of neovascularization, besides drugs that can block this growth, we need techniques capable of measuring the functioning of this drug, realizing if it is able to reduce neovascularization or delay it, and also analyzing the patient before treatment to reach a scale of the disease has progressed. In addition, the visualization of the contours of the tumor are difficult interpretations by the specialists due to the low visibility in imaging examinations [18], emphasizing once again the necessity of more precise techniques. The Box-Counting technique, used to quantify the fractal dimension by analyzing complex patterns where it divides a set of data into smaller scales, proves the great possibilities of aid to the diagnosis, becoming a potential ally for the improvement and development of computational systems by image recognition $[19,20]$. Computational tests suggest that the fractal model presented satisfactory sensitivity in the processing of the mammographic images for the detection of clusters (cluster of sectors that constitutes the smallest unit of allocation able to be addressed on a magnetic disk) of microcalcifications, making it possible to accurately classify the mammary tissue with benign tumors, differentiating them from malignant tumors, highlighting their low cost [21].

The results indicated that the fractal analysis is an advantageous method applicable to health equipment, strengthening the development of systems that seek to prioritize the early identification of neoplasia. It also contributes to the diagnosis in the process of image formation, having as a differential the elimination of disturbing factors in the image, accelerating the time necessary for the diagnosis to close and also contributing to the follow-up and development of the neoplasia. Already published studies used the fractal dimensions in the differentiation of malignant and benign tumors, the first step that was analyzed was the edges found in the images. The obtained results were evaluated to define from certain reference values, if the analyzed tumors are benign or malignant. In addition, the fractal dimensions were also used for segmentation, analysis and classification of skin lesions [15,22] (Figures 4 and 5).

Coupled with the Box-Counting method, the study of the eccentricity of ellipses is obtained by means of calculations to measure their flattening. The closer to the value 1 (one), the ellipse tends to be more pressed, and the closer to 0 (zero) to the shape of a circumference. Through the study of the eccentricity of the ellipse, it is possible to have more precision about the determination of the type of tumor analyzed, this is due to the fact that the more circular the tumor the more chances
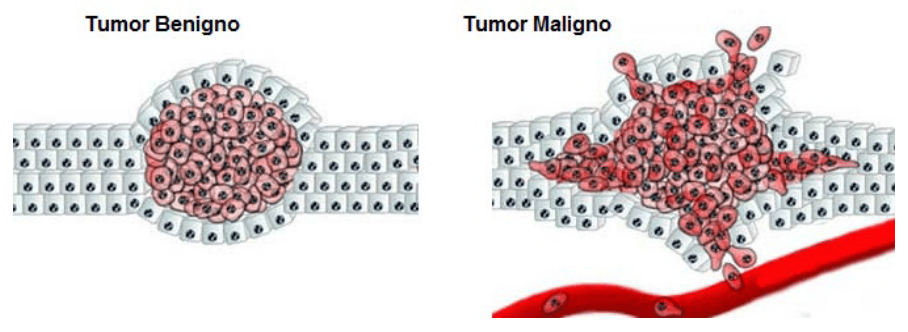

Figure 4. Transition of mesenchymal epithelium in neoplastic process

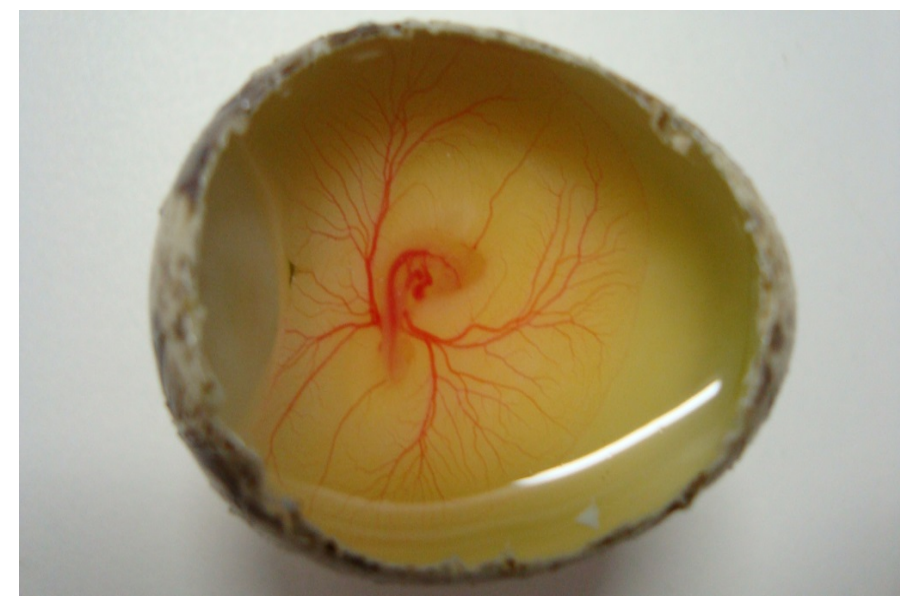

Figure 5. Vascularization and angiogenesis study using quail embryos (Coturnix coturnix) 
of being benign, and the more elliptical, the more chances of be malignant [23].

\section{Conclusion}

Faced with the great need to improve the detection of cancer, its progression and development of the treatment, to have a breakthrough in individual medicine, the use of image processing has been gaining more and more space, and is an area of great prosperity. Research has already shown that fractal dimensions can be used to aid in the diagnosis of breast, oral and retinopathy. These studies show how important are studies related to image processing and the use of technology, aiming at improvements in methods and procedures in the medical field.

\section{References}

1. Datasus (2018) Departamento de Informática do SUS; Ministério da Saúde, Sistema de Informações sobre Mortalidade- SIM e base demográfica do IBGE. Disponível em.

2. OPAS (2019) Organização Pan Americana de Saúde; WHO - World Health Organization. Folha Informativa-Câncer. OPAS; OMS. Disponível em: Acesso em.

3. INCA (2019) National Cancer Institute José de Alencar. Números de Câncer. Disponível em: Acesso em.

4. Pinho MSL (2005) Angiogenesis: the proliferative trigger. Revista Brasileira de. Disponível em.

5. Yuan Y, Jiang YC, Sun CK, Chen QM (2016) Role of the tumor microenvironment in tumor progression and the clinical applications. Oncology reports 35: 2499-2515.

6. Onuchic AC, Chammas R (2010) Cancer and the tumor microenvironment. Revista de Medicina 89: 21-31.

7. Hanhan Douglas, Weinberg RA (2000) The hallmarks of cancer. Cell 100: 57-70.

8. Hanhan D, Weinberg RA (2011) Hallmarks of cancer: the next generation. Cell 144: 646-674.

9. Graca B, Carla L, Ana SC, Gisela M, Paulo F, et al. (2004) ANGIOGÉNESE E CANCRO da biopatologia à terapêutica. Acta Médica Portuguesa 17: 76-93.
10. Ramjia W, Rakesh R, Griffioen Arjan W, Duda DG (2017) Anti-angiogenesis for cancer revisited: Is there a role for combinations with immunotherapy? Angiogenesis 20: $185-204$.

11. DA Silva, Míriem M, De Souza, Wallysonn A (2010) Dimensão Fractal. Revista Eletrônica de Matemática 1: 2.

12. De Medeiros, R Dom Manuel (2019) Introducing fractal geometry in middle school: an approach based on the forms of objects constructed by nature.

13. Assis TA, Vivas Miranda JG, Mota FD, Andrade RF, Castilho CM (2008) Fractal geometry: properties and features of ideal fractals. Revista Brasileira de Ensino de Física 30: 2304-2310.

14. Backes AR, Bruno OM (2005) Fractal dimension estimation techniques: a comparative study. Infocomp 4: 50-58.

15. Padilha Renata Junges (2017) Computational methods in the differentiation of breast tumors. Gedecon Magazine-Management and Development in Context 5:17-20.

16. Ng CF, Frieboes HB (2017) Model of vascular desmoplastic multispecies tumor growth. J Theor Biol 430: 245-282.

17. Carvalho CI C de (2016) Use of mice in the study of new targets of anti-angiogenic oncology therapy, Universidade de Lisboa.

18. Ribeiro PB (2006) Classification by contour analysis of breast nodules using artificial neural networks. Tese de Doutorado. Universidade de São Paulo.

19. Sarkar N, Chaudhuri BB (1994) An efficient differential box-counting approach to compute fractal dimension of image. IEEE 24: 115-120.

20. Li J, Sun C, Du Q (2006) A new box-counting method for estimation of image fracta dimension. In2006 International Conference on Image Processing. IEEE 1: 3029-3032.

21. Goda Rúpila Rami da Silva (2016) Computational intelligence applied in mammary microcalcifications, Universidade Federal Rural do Rio de Janeiro.

22. Fabri TA (2015) Segmentation, analysis and classification of images of skin lesions using fractal dimension. Federal Technological University of Paraná.

23. De M, Rafael HC, Conci A (2013) How succolarity could be used as another fractal measure in image analysis. Telecommunication Systems 52: 1643-1655.

Copyright: (C2019 da Silva Júnior JG. This is an open-access article distributed under the terms of the Creative Commons Attribution License, which permits unrestricted use, distribution, and reproduction in any medium, provided the original author and source are credited. 